This article was downloaded by: [The University of Manchester]

On: 6 May 2011

Access details: Access Details: [subscription number 932638668]

Publisher Psychology Press

Informa Ltd Registered in England and Wales Registered Number: 1072954 Registered office: Mortimer House, 3741 Mortimer Street, London W1T 3JH, UK

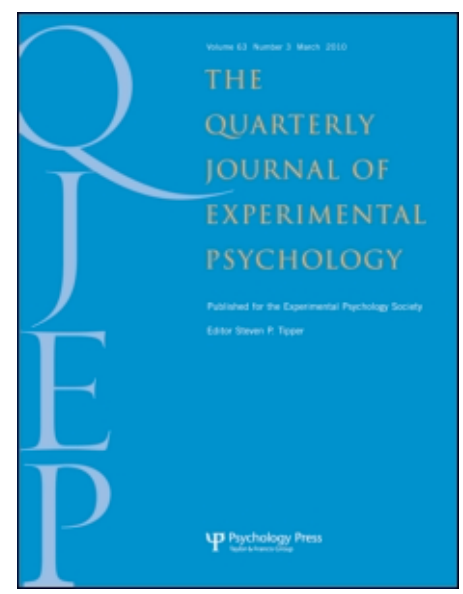

\title{
The Quarterly Journal of Experimental Psychology
}

Publication details, including instructions for authors and subscription information:

http://www.informaworld.com/smpp/title content=t716100704

\section{More is still not better: Testing the perturbation model of temporal reference memory across different modalities and tasks}

Ruth S. Ogden ${ }^{\text {a }}$ Luke A. Jones ${ }^{\mathrm{a}}$

${ }^{\text {a }}$ The University of Manchester, Manchester, UK

First published on: 10 September 2008

To cite this Article Ogden, Ruth S. and Jones, Luke A.(2009) 'More is still not better: Testing the perturbation model of temporal reference memory across different modalities and tasks', The Quarterly Journal of Experimental Psychology, 62: 5, 909 - 924, First published on: 10 September 2008 (iFirst)

To link to this Article: DOI: $10.1080 / 17470210802329201$

URL: http://dx.doi.org/10.1080/17470210802329201

\section{PLEASE SCROLL DOWN FOR ARTICLE}

Full terms and conditions of use: http://www.informaworld.com/terms-and-conditions-of-access.pdf

This article may be used for research, teaching and private study purposes. Any substantial or systematic reproduction, re-distribution, re-selling, loan or sub-licensing, systematic supply or distribution in any form to anyone is expressly forbidden.

The publisher does not give any warranty express or implied or make any representation that the contents will be complete or accurate or up to date. The accuracy of any instructions, formulae and drug doses should be independently verified with primary sources. The publisher shall not be liable for any loss, actions, claims, proceedings, demand or costs or damages whatsoever or howsoever caused arising directly or indirectly in connection with or arising out of the use of this material. 


\title{
More is still not better: Testing the perturbation model of temporal reference memory across different modalities and tasks
}

\author{
Ruth S. Ogden and Luke A. Jones \\ The University of Manchester, Manchester, UK
}

\begin{abstract}
The ability of the perturbation model (Jones \& Wearden, 2003) to account for reference memory function in a visual temporal generalization task and auditory and visual reproduction tasks was examined. In all tasks the number of presentations of the standard was manipulated (1, 3, or 5), and its effect on performance was compared. In visual temporal generalization the number of presentations of the standard did not affect the number of times the standard was correctly identified, nor did it affect the overall temporal generalization gradient. In auditory reproduction there was no effect of the number of times the standard was presented on mean reproductions. In visual reproduction mean reproductions were shorter when the standard was only presented once; however, this effect was reduced when a visual cue was provided before the first presentation of the standard. Whilst the results of all experiments are best accounted for by the perturbation model there appears to be some attentional benefit to multiple presentations of the standard in visual reproduction.
\end{abstract}

Keywords: Temporal reproduction; Temporal generalization; Reference memory; Modality.

The comparative temporal processing of stimuli of different modalities has been the focus of numerous studies (e.g., Goldstone \& Lhamon, 1974; Klapproth, 2002; Noulhiane, Pouthas, \& Samson, in press; Penney, Gibbon, \& Meck, 2000; Sebel \& Wilsoncroft, 1983; Wearden, Edwards, Fakhri, \& Percival, 1998; Wearden, Todd, \& Jones, 2006). Typically these studies compare performance between trials using auditory stimuli with those using visual stimuli. Alternatively, the effects of manipulations such as drug intake or increased memory load are compared in both the auditory and visual domains. Manipulations tend to have the same general effect on both auditory and visual stimuli-for example, when age (Droit-Volet \& Wearden, 2001), drug intake (Meck, 1983), lesions to the brain (Meck, 1988; Meck, Church, Wenk, \& Olton, 1987; Olton, 1989), and existing memory content (Grondin, 2005) are altered. When the focus of the study is to compare the performance on auditory and visual tasks two performance differences are consistently reported: Auditory stimuli are perceived as lasting for

Correspondence should be addressed to R. S. Ogden, School of Psychological Sciences, The University of Manchester, M13 9PL, UK. E-mail: Ruth.Ogden@postgrad.manchester.ac.uk

The work reported in this article was conducted as part of the $\mathrm{PhD}$ research of the first author. 
longer than visual stimuli of the same actual duration (Goldstone \& Lhamon, 1974; Wearden et al., 1998; Wearden et al., 2006) and are perceived more accurately than visual stimuli-that is, performance on auditory tasks is less variable than that on visual tasks (Collier \& Logan, 2000; Goldstone, Boardman, \& Lhamon, 1959; Wearden et al., 1998; Wearden, et al., 2006).

Whilst a number of studies have explored the possible reasons for these modality differences the root cause remains unclear. Wearden et al. (1998) suggested that the internal clock may run at a faster rate for auditory stimuli than for visual stimuli. A faster clock speed would result in a larger number of "ticks" being accumulated in a given period, accounting for auditory stimuli being perceived as longer than visual stimuli. This explanation is also consistent with the fact that the modality difference between auditory and visual verbal estimation manifests itself in a slope effect, in which the difference in the subjective duration of auditory and visual stimuli increases with increasing duration, indicative of a multiplicative process.

Penney et al. (2000) suggest that in addition to differences in clock speed the way intervals are stored in reference memory may also contribute towards modality differences as modality effects are generally only reported in within-subject experiments. Penney et al. (2000) suggested that when a duration (for example the standard in a temporal generalization task) is presented in both modalities the two representations of the duration will mix in reference memory. The value stored as the standard will therefore fall somewhere inbetween the value for the faster clock speed of the auditory stimuli and the slower clock speed of the visual stimuli. As a result of this mixing all subsequent auditory comparisons of the same actual duration as the standard will be judged as longer than the value in reference memory, and all visually presented comparisons of the same actual duration will be judged as shorter.

Wearden et al. (2006) tested the predictions of the memory mixing model by comparing withinsubject and between-subjects experiments. They found that in the between-subjects experiments in which participants received and were tested on only one modality a slope effect between auditory and visual performance of the same magnitude as that observed in the within-subject experiment was still found. This suggests that memory mixing is not a necessary condition for modality differences to occur, though it may be a sufficient one.

The reason for the relatively higher variability in the timing of visual stimuli is somewhat clearer. Wearden et al. (1998) point out that the slower clock speed of visual stimuli would produce more variable performance; however, they suggest that a more likely cause of increased variability in visual timing is a difference in the variability of the switch onset-offset latency. The switch connects the pacemaker with the accumulator in the information processing model of SET (scalar expectancy theory; Church, 1984; Gibbon, 1977). For a duration to be timed the switch must close at the onset of the stimulus allowing pulses to flow into the accumulator. When the stimulus being timed ends the switch opens, and accumulation ceases. For accurate timing the onset and offset latencies of the switch must be the same or very similar. More variable timing may occur if the closure of the switch is slower than the opening or vice versa, or if the onset-offset latency changes between trials. If the switch latency of the onset and offset of the switch are similar or identical then the actual duration of the latency is of no consequence to accurate performance; however, if they differ significantly then the duration will be underestimated (if onset latency is greater than offset) or overestimated (if offset latency is greater than onset). For a more detailed exposition of switch processes see Wearden et al. (1998). Wearden et al. (1998) suggested that when pacemaker speed was increased (by presenting a train of clicks prior to the presentation of the standard) switch latency differences in modality would become more apparent as pacemaker variability would be reduced. Their Experiment 3 confirmed this suggestion; variability in mean verbal estimates remained higher for visual stimuli than for auditory stimuli even when pacemaker speed was 
increased. The switch may be more variable when timing visual stimuli than auditory stimuli because auditory stimuli are easier to process or more automatically attended to than visual stimuli.

A series of papers by Penney and colleagues also suggest that switch operation may play a fundamental role in modality differences in timing (Penney, 2003; Penney et al., 2000; Penney \& Tourret, 2005). Unlike Wearden et al. (1998), Penney suggests that the switch oscillates between being opened and closed through the timing of a duration. This idea is similar to the earlier proposed attentional gate model of Zakay and Block (1997), in which the gate can open more widely when more attention is paid to a task resulting in faster accumulation (for further discussion of attention in timing and the attentional gate theory, see Fortin, 1999; Fortin \& Breton, 1995; Fortin \& Rousseau, 1987, 1998; Fortin, Rousseau, Bourque, \& Kirouac, 1993; Lejeune, 1998, 2000; Zakay, 2000). The amount of oscillation is determined by the amount of attention paid to the stimulus. Penney suggests that modality differences therefore occur because the switch oscillates relatively less when the stimuli is auditory than when it is visual. This is thought to occur because it is easier to maintain the switch in a closed position when the stimulus is auditory (Penney, 2003). This idea was supported by Droit-Volet, Meck, and Penney (2007) who attributed a larger modality effect in children to children's reduced ability to focus attention in comparison with adults.

Easier or more fluent processing of auditory stimuli would account for increased variability in visual tasks and superior performance in auditory tasks (Collier \& Logan, 2000; Glenberg \& Jona, 1991). Increased fluency in auditory processing is demonstrated not only by reduced reaction times to auditory stimuli (Jaskowski, Jaroszyk, \& Hogan-Jazierska, 1990), but also by the dominance of auditory stimuli when a duration is presented as a compound of auditory and visual stimuli; Walker and Scott (1981) found that the duration of a tone and light presented simultaneously was judged to be more similar to that of a tone of the same duration than that of a light of the same duration, suggesting auditory dominance in the perception of compound tones. A similar effect was observed by Penney et al. (2000) who suggested that a memory representation will be dominated by the auditory representation when both auditory and visual stimuli have been presented. Ogden, Wearden, and Jones (2008), however, suggest that visual temporal reference memory may be superior to auditory temporal reference memory in that memory for visual durations are less vulnerable to interference, and visual reference memory appears to have a greater capacity than auditory temporal reference memory.

One way to test whether auditory stimuli are more readily processed than visual stimuli is to investigate whether performance accuracy in both modalities is affected by the number of opportunities to learn a duration. In a temporal generalization task, for example, increasing the number of presentations of the standard will provide more opportunity for the standard to be encoded, presumably leading to more accurate storage if initial encoding is effortful or erroneous. Previous research in this area has typically examined the effect of increasing the number of opportunities to learn a duration during rhythm perception or repetitive tapping performance; alternatively, comparisons have been made between musicians and nonmusicians (e.g., Drake \& Botte, 1993; Ivry \& Hazeltine, 1995; McAuley \& Kidd, 1998). Ivry and Hazeltine, for example, compared accuracy on repetitive or isolated rhythm tapping and found that durationdependent variability was reduced in repetitive compared with isolated tapping.

The effect of the number of times a standard is presented has more recently been investigated by Jones and Wearden (2003) using auditory stimuli in a temporal generalization task. Jones and Wearden developed a "changing standards" version of the temporal generalization task in which a new standard was presented at the start of each trial (similar tasks have previously been developed for use with animals by Rodriguez-Gironés \& Kacelnik, 1999). During Jones and Wearden's task each standard was presented either one, three, or five times. Participants were then required to 
recognize the standard from an array of comparison durations. Increasing the number of times the standard was presented had no effect on performance, in that correct identifications of the standard did not increase, and response variability did not decrease as the number of presentations increased. The differing findings of Jones and Wearden (2003) and Ivry and Hazeltine (1995) could be explained by the fact that in Ivry and Hazeltine's multiplepresentations condition participants were required to synchronize their tapping to a rhythm for 12 productions of the rhythm and then were required to tap independently; therefore not only did they have more experience of hearing the duration but they also had more experience reproducing it. It is therefore possible that the opportunity to practise reproducing the duration resulted in a reduction in duration-dependent variability rather than the increased opportunity to learn the duration.

Jones and Wearden (2003) were interested in why increasing the number of opportunities to learn a duration did not improve performance, and to explain this they examined how multiple durations were stored in reference memory. Prior to 2003 two main models of reference memory function existed: the sampling (SAM) and the averaging model (AVE; for discussion of the origins of these models, see Brunner, Fairhurst, Stolovitsky, \& Gibbon, 1997; Gibbon, Church, Fairhurst, \& Kacelnik, 1988; Jones \& Wearden, 2003). Jones and Wearden used computer modelling to assess whether either of these models could account for this finding that temporal task performance does not appear to improve with increased opportunities to learn a duration. Jones and Wearden modelled temporal generalization performance based on the predictions of the two models whilst manipulating the number of times the standard duration was presented.

The AVE model of reference memory function suggests that repeated presentations of a standard are averaged together in reference memory, resulting in the standard becoming the arithmetic mean of all encoded presentations of the standard; in a trial this mean value is used for all future judgements. The idea that participants can average durations in reference memory was previously suggested by Schulze (1989) and demonstrated by Wearden and Jones (2007) in a task in which participants were instructed to average all presentations of the standard. The AVE model was, however, unable to accurately embody temporal generalization performance as observed by Jones and Wearden (2003) as its predictions led to an increase in the mean proportion of yes responses at the standard as the number of presentations of the standard increases.

The sampling account of reference memory function is an embodiment of the ideas incorporated into SET's reference memory (see Brunner et al., 1997; Gibbon et al., 1988; Jones \& Wearden, 2003, for a discussion). The SAM account of reference memory function suggests that repeated presentations of a standard are stored individually, and, when required, one is selected at random from the array for use in a trial. Modelling by Jones and Wearden revealed that the predictions of the SAM model suggest that increasing the number of presentations of the standard does not affect the number of correct identifications of the standard. Although this might appear to accommodate the findings of Jones and Wearden (2003), the SAM model cannot accommodate the rapid transfer between large changes in reinforcement values observed in animal timing (see Lejeune, Ferrara, Simons, \& Wearden, 1997, for a discussion) and also the slow transitions for small changes, as in drug manipulation studies (Meck, 1983) as the contents of reference memory are sampled at random.

The inability of either SAM or AVE to accommodate data from both human and animal timing led Jones and Wearden to develop the perturbation model of temporal reference memory function. The perturbation model suggests that after an item is presented and transformed by $K^{*}$ (a Gaussian distribution with a mean of 1 , see Jones \& Wearden, 2003, for a full account) it is stored in reference memory as distribution with an upper and lower bound, rather than a single item or a succession of very similar items. The bounds of the distribution are proportional to the duration stored. If any subsequent entries into reference memory fall outside of the bounds

\section{THE QUARTERLY JOURNAL OF EXPERIMENTAL PSYCHOLOGY, 2009, 62 (5)}


a new entry is made; this entry then perturbs the existing entry and is used for all future comparisons. If future entries fall within the bounds then no new entry is made; the distribution remains the same, and no new representations are formed. The perturbation model can account for the findings of Jones and Wearden because it does not predict any change in responding as the number of presentations of the standard increases. It is also able to accommodate the relatively fast transition times observed with large changes in reinforcement time because they will perturb the model quickly and the relatively slow changes in responding associated with small changes in reinforcement time because they will shift the distribution slowly.

Whilst it would appear that the perturbation model is better able to account for human temporal generalization performance than the SAM or the AVE, it has only ever been tested in one modality on one temporal task. This study therefore examines whether reference memory functions in the same way for different modalities. Perhaps more importantly this study also investigates whether the characteristics of reference memory function are the same across different temporal tasks. The experiments in this study therefore initially replicate the methodology of Jones and Wearden (2003, Exp. 3) using visual stimuli (Experiment 1). Experiments 2, 3, and 4 then examine reference memory function on different temporal tasks by employing a reproduction-based task using visual and auditory stimuli. During a reproduction task participants are presented with a standard duration and are then required to reproduce its duration. Reproductions typically take the form of terminating the sounding of a tone by pressing a key when the tone has lasted for the same duration as the standard (Noulhiane et al., in press; Wearden, 2003; Woodrow, 1930). Reproduction relies on accurate recall of the standard rather than accurate recognition as in temporal generalization. Reproduction may be a stronger test of the perturbation model as it may be the case that the effect of rehearsal (multiple presentations) only leads to enhanced performance in a recall-type task.
The aims of this study were therefore threefold. First it investigated whether durations of different modalities are encoded into reference memory in the same way, thus testing the perturbation model in modalities other than auditory. Secondly, the perturbation model's ability to account for performance on non-recognitionbased tasks was assessed using auditory and visual reproduction tasks. Finally the results of all four experiments and those of Jones and Wearden (2003) were compared to assess whether auditory stimuli are in fact easier to encode and process than visual stimuli.

\section{EXPERIMENT 1}

Experiment 1 constituted a near replication of Jones and Wearden's (2003) Experiment 3 but with visual stimuli instead of auditory stimuli. The changing-standards temporal generalization method was employed, in which participants received a standard (presented 1, 3, or 5 times) in the form of a blue square on a white background. Following the standard a number of comparisons were presented, and the duration of each was compared to that of the standard. Comparisons were shorter, longer, or the same duration as the standard.

The absence of performance improvement on visual temporal generalization task as the number of presentations of the standard increased would confirm that visual and auditory stimuli are stored in the same general way in reference memory; in other words the perturbation model is applicable to both modalities. Alternatively an improvement in performance with increased presentation would cast doubt on the generality of the perturbation model outside of the auditory domain.

\section{Method}

\section{Participants}

A total of 17 University of Manchester students were paid $£ 5$ for participating. 


\section{Apparatus}

An IBM PC-compatible computer recorded all experimental events. The stimuli were presented on the computer screen in the form of a blue square $4.5 \mathrm{~cm} \times 4.5 \mathrm{~cm}$ on a white background. The keyboard measured participants' responses. The program used to run the experiment and record data was written in E-prime (Psychology Software Tools, Inc., Pittsburgh, PA).

\section{Procedure}

The changing-standards variant of temporal generalization was employed. Participants were told that they would be presented with the standard duration. The standard was a blue square $4.5 \mathrm{~cm} \times 4.5 \mathrm{~cm}$ presented on a white background. The duration of presentation was drawn from a uniform distribution ranging from 400$800 \mathrm{~ms}$. Depending on the testing condition the standard was presented 1,3 , or 5 times. All presentations were the same duration. A delay, the duration of which was drawn from a uniform distribution ranging from 1,500-2,000 ms, was imposed between presentations of the standard. Following the presentation of the standard participants received comparison blue squares whose duration was the standard (whatever it was on that block) multiplied by $0.4,0.6,0.8,1,1.2,1.4$, and 1.6 with the order randomized. After the comparison stimulus presentation, the participant judged whether or not the stimulus had the same duration as the standard, making a "Y" (Yes) or "N" (No) response on the keyboard. No feedback as to response accuracy was given. Following presentation of all seven comparison stimuli, a new standard was randomly generated for the next block and so on. Participants had been informed by previous instructions that the standard would change for each block.

In total there were 51 trials, 17 in each testing condition (1, 3, or 5 presentations of the standard). The number of times the standard was presented on each block was random. Participants completed all trials in one session; the experiment was selfpaced but lasted for approximately 30 minutes.

\section{Results}

Figure 1 shows temporal generalization gradients-the mean proportion of YES responses (identification of a presented comparison duration as the standard) plotted against the comparison/ standard ratio for the three testing condition (1, 3, or 5 presentations of the standard). Inspection of the data in Figure 1 suggests that in all testing conditions peak responding is at or around the standard; there appears to be no improvement in performance as the number of presentations of the standard increases. This suggestion was confirmed by the statistical analysis.

An overall repeated measures analysis of variance (ANOVA) also used number of standard presentations (1, 3, or 5) and comparison/standard ratio (effectively the duration of the comparison) as within-subject factors. There was no effect of number of standard presentations, $F(2,34)=$ $1.12, p=.34$, but there was a significant effect of comparison/standard ratio, $F(1.84,97.19)=18.10$, $M S E=0.14, p<.01$ (Greenhouse-Geisser corrected), indicating that participants were sensitive to comparison duration. There was no number of standard presentations by comparison/standard ratio interaction, $F<1$, indicating that the number of presentations of the standard did not

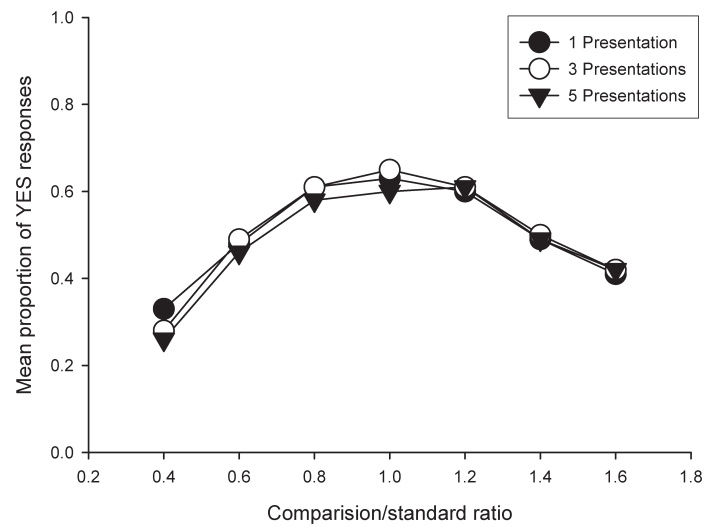

Figure 1. Temporal generalization gradients (proportion of YES responses-identifications of a comparison duration as the standard-plotted against comparison/standard ratio) from Experiment 1. Data are shown separately for 1 (filled circle), 3 (unfilled circle), and 5 (filled triangle) presentations of the standard. 
significantly affect the shape of the temporal generalization gradients.

\section{Discussion}

The results of this experiment did not reveal any effect of the number of presentations of the standard on the accuracy of identification of the standard when it was presented, the overall level of YES responses, or the shape of the temporal generalization gradients. As such the results of this experiment mirror those of Jones and Wearden (2003) in that the number of presentations of the standard does not affect temporal generalization performance. The absence of an effect of multiple presentations of the standard supports the perturbation model of reference memory function for visual as well as auditory stimuli.

A potential criticism of the method used in this Experiment 1 and Jones and Wearden (2003) is that temporal generalization may not show the beneficial effect of multiple opportunities to encode the standard as it is a recognition task. Perhaps a more suitable task would be reproduction. In a reproduction task participants are required to reproduce a presented standard, usually by pressing a button after the duration of the standard had passed. Reproduction relies solely on the participant's memory of the standard and is not a judgement of similarity between two items like temporal generalization. It is therefore possible that increased exposure to a standard in a reproduction task may lead to an increase in performance accuracy, unlike in temporal generalization. It is also possible that reference memory may operate in different ways on different temporal tasks depending on the demands of each task.

\section{EXPERIMENT 2}

Experiment 2 examined whether the perturbation model can account for reference memory function on tasks other than temporal generalization. In Experiment 2 participants performed either an auditory or a visual reproduction task. Participants were therefore presented with either a $500-\mathrm{Hz}$ tone or a blue square as a standard and were then asked to reproduce its duration by terminating either a subsequent tone or the presence of a subsequent blue square. As in Experiment 1 the standard was presented 1,3 , or 5 times.

\section{Method}

\section{Participants}

A total of 37 University of Manchester students participated for course credit. They were allocated to one of two testing conditions (auditory 20 participants, visual 17 participants).

\section{Apparatus}

The apparatus was as that in Experiment 1 with the addition of a serial response box used to terminate reproductions.

\section{Procedure}

A variant of the temporal reproduction method was used. Participants were instructed that they would be presented with a standard and that their task was to reproduce the duration of the standard by terminating either a tone in the auditory condition or the presence of a blue square in the visual condition. The number of times the standard was presented (1, 3, or 5 times) was varied in line with Experiment 1. The following procedure outlines the task for the auditory condition. For the visual condition all experimental details remain identical; however, the stimuli were presented as blue squares $4.5 \mathrm{~cm} \times 4.5 \mathrm{~cm}$.

Participants were given three initial practice trials followed by the experiment. At the beginning of each trial participants were presented with the standard, which was a $500-\mathrm{Hz}$ tone. The following durations were used for the standard: $110 \mathrm{~ms}, 199 \mathrm{~ms}, 241 \mathrm{~ms}, 352 \mathrm{~ms}, 401 \mathrm{~ms}, 492 \mathrm{~ms}$, $512 \mathrm{~ms}, 563 \mathrm{~ms}, 666 \mathrm{~ms}, 712 \mathrm{~ms}, 809 \mathrm{~ms}, 844 \mathrm{~ms}$, $916 \mathrm{~ms}, 1,071 \mathrm{~ms}$, or $1,141 \mathrm{~ms}$. Each duration was used once in each of the three testing conditions $(1,3$, or 5 presentations of the standard) giving a total of 45 experimental trials. The order of trials was randomized for each participant. On trials where the standard was 
presented more than once a delay was imposed between each presentation, the duration of which was drawn from a uniform distribution ranging from 1,500-2,000 ms. After the presentation of the standard there was a delay, the duration of which was drawn from a uniform distribution ranging from 2,000-5,000 ms. Following the presentation of the standard participants were instructed to reproduce the duration of the standard by terminating the sounding of a tone by pressing Button 1 on a serial response box. A $500-\mathrm{Hz}$ tone would then sound until the participant terminated it. Participants were then instructed that a new standard was to be presented. No performance feedback was provided. Participants received all trials in one experimental session lasting approximately 25 minutes.

\section{Results}

Data were excluded from analysis when the reproduction was greater than 2 standard deviations above or below the mean. In total data from 32 of the 1,665 trials were excluded (15 auditory, 17 visual).

Figure 2 shows mean reproductions for each of the three testing conditions $(1,3$, or 5 presentations of the standard) for both the visual and the auditory testing groups. For the auditory group (upper panel), comparison of performance on the three testing conditions (1, 3 , or 5 presentations) shows that whilst superimposition was not perfect there is no systematic difference in performance across the testing conditions. For the visual group (lower panel), reproductions appear significantly shorter when the standard is only presented once than when it is presented three or five times. There appears to be no difference in the duration of reproductions when the standard was presented three or five times. For both testing groups, as is typical of reproduction data, participants appear to overproduce the duration of the shorter standards and underproduce the duration of the longer standards.

Linear regressions were carried out on individual participants' data giving slope and intercept values for each of the three testing conditions.
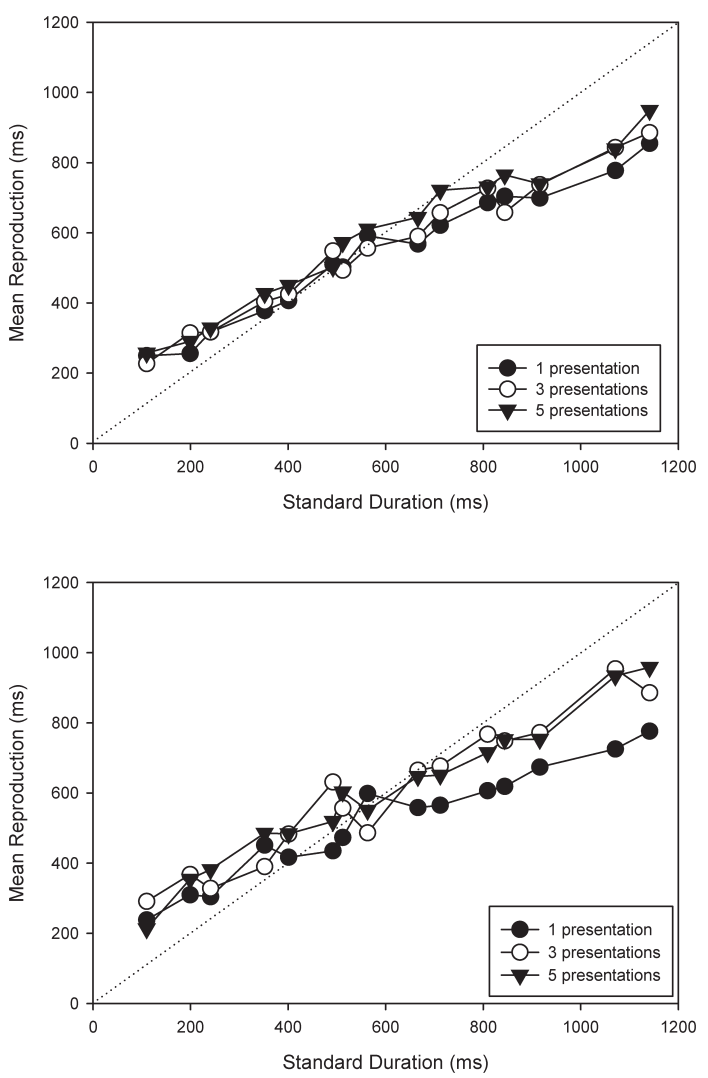

Figure 2. Mean reproductions from Experiment 2, plotted against stimulus duration in ms. Data are shown separately for 1 (filled circle), 3 (unfilled circle), and 5 (filled triangle) presentations of the standard. Upper panel shows data from the auditory condition; lower panel shows data from the visual condition.

Repeated measures ANOVAs compared slope and intercept values for the three testing conditions (1, 3 , or 5 presentations) across the two testing groups (modality; auditory and visual).

For intercept values there was no significant effect of modality, $F<1$, nor was there an effect of the number of presentations of the standard, $F<1$. There was also no significant interaction between the number of presentations of the standard and modality, $F(2,72)=2.85, M S E=7,586, p=.65$.

For the slope of the function there was a significant main effect of the number of presentations of the standard, $F(2,72)=5.48, M S E=0.027$, $p<.01$. There was also a significant interaction 
between the number of presentations of the standard and the modality of presentation, $F(2$, $72)=5.10, M S E=0.027, p<.01$. There was no significant main effect of modality, $F<1$. Examination of Figure 3 demonstrates that the slope of the function was considerably flatter when the standard was only presented once and when it was visual than when the standard was presented three or five times, or once when visual.

Analysis of the slope of the function in the visual condition alone confirmed a significant effect of the slope of the function, $F(2,34)=6.79$, $M S E=0.027, p<.01$. Planned comparisons revealed a significant difference between the slope of the functions of 1 and 3 presentations, $F(1,17)=8.34, M S E=0.027, p<.01$, and 1 and 5 presentations, $F(1,17)=8.71, M S E=$ $0.027, p<.01$. There was no significant difference between the slope of the functions of 3 and 5 presentations, $F<1$. Analysis of the slope of the function in the visual condition alone confirmed no effect of the number of presentation of the standard on the slope of the function.

\section{Discussion}

Experiment 2 suggests that increasing the number of times a standard is presented affects the

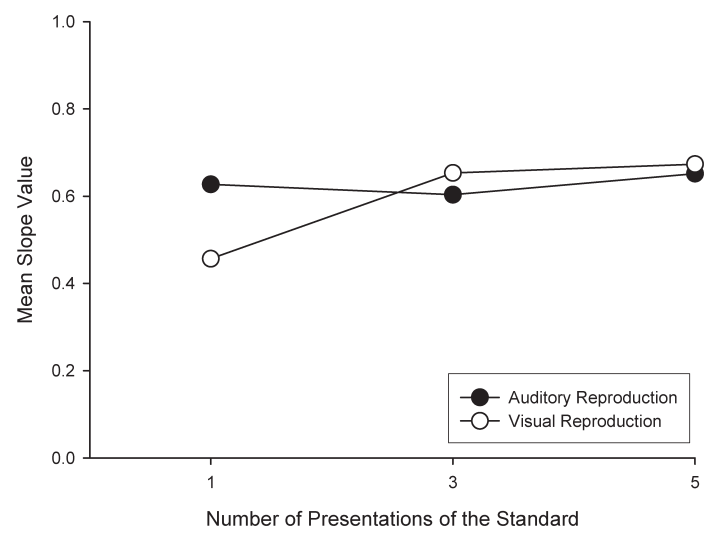

Figure 3. The mean slope values derived from linear regressions of participants' reproductions in the 1, 3, and 5 presentation conditions of Experiment 2. Auditory reproduction and visual reproduction conditions are shown separately. duration of reproductions for visual but not auditory stimuli. For auditory stimuli, the number of times a standard was presented did not have a significant effect on the participant's reproductions of the standard. The absence of any beneficial effect of multiple presentations of the standard supports the perturbation model of temporal reference memory in auditory nonrecognition-based tasks. The reproductions from both the auditory and the visual groups exhibit the classic pattern of convention to Vierordt's Law, in which short durations are overestimated, and long durations are underestimated. To our knowledge this effect has not been demonstrated intermodally to date.

For visual stimuli, reproductions were shorter when the standard was only presented once than when it was presented visually three or five times or when presented as auditory stimulus one, three, or five times. Examination of Figure 3 and planned comparisons demonstrate that for visual stimuli there is no difference in reproduction duration when the number of presentations of the standard is increased from three to five. It would therefore seem that for visual reproduction there may be some effect of receiving a standard more than once, primarily an increase in the duration of the reproductions when the standard is presented more than once. Increasing the number of presentations beyond three does not, however, affect the duration of reproductions.

At first glance the result from the visual condition does not appear compatible with the perturbation model's explanation of reference memory function due to the performance differences when the standard is presented once in comparison to three or five times. The averaging model is also unable to account for the results as it would also predict differences between three and five presentations, an effect not observed in these data. As neither model immediately appears to be able to accommodate this finding, the obvious question becomes why would there be an effect of increasing the number of presentations beyond one, but not beyond that? One possible answer is that in trials where the standard is presented more than once the 
first presentation acts as an attentional cue for subsequent presentations.

On trials where the standard is presented only once participants may fail to fully pay attention to the standard, resulting in them missing the start of its presentation. Missing a proportion of the standard would make the memory representation shorter and would therefore make reproductions shorter. On trials where the standard is presented more than once, the first presentation may be used as an attentional cue to the arrival of subsequent presentations, allowing the entire presentation of subsequent standards to be encoded, rather than the start portion being missed. Encoding the whole duration of the standard, rather than most of it, will lead to a longer representation being stored in reference memory; this longer representation is therefore likely to perturb the existing representation made when the standard was only presented once, leading to longer reproductions. Further presentations beyond the second one would be unlikely to perturb the existing representation and would therefore not lead to performance differences. The perturbation model therefore also seems able to account for visual reproduction performance if we accept that there is some attentional benefit to multiple presentations of the standard.

Experiment 3 was designed to examine whether a lack of attention to the first presentation of the standard is the cause of shorter reproductions when the standard is only presented once by providing an attentional cue prior to the first presentation of the standard. The idea of attentional cues being beneficial in timing is not new. Droit-Volet (2003) found that providing children with a cue to the start of a stimulus duration in a visual temporal discrimination task led to an increase in the proportion of long responses. Attentional cues are likely to be more beneficial in visual timing for two reasons; one is that unlike auditory stimuli visual stimuli must be actively attended to if their start and end is to be encoded accurately. Secondly, the operation of the switch is thought to be more variable in visual timing than in auditory timing (Penney, 2003; Wearden et al., 1998).

\section{EXPERIMENT 3}

Experiment 3 investigated the potential benefit of an attentional cue prior to the first presentation of the standard. A visually presented 3, 2, 1 countdown was be presented prior to the first presentation of the standard. It was anticipated that the use of a cue that is the same modality as the standard and that occurs in the same spatial location was likely to improve attention to the first presentation of the standard.

\section{Method}

\section{Participants}

A total of 17 University of Manchester students participated for course credit.

\section{Procedure and apparatus}

The procedure was essentially a replication of the visual condition of Experiment 2 with the provision of an attentional cue in the form of a 3,2 , 1 countdown prior to the presentation of the first standard on each trial. On each trial participants were informed that they were about to the presented with the standard. A period of $1,500 \mathrm{~ms}$ before the presentation of the standard the number 3 was presented on the screen for $250 \mathrm{~ms}$; there was then a break of $250 \mathrm{~ms}$ followed by the presentation of the number 2 for $250 \mathrm{~ms}$, then a break of $250 \mathrm{~ms}$ followed by the presentation of the number 1 for $250 \mathrm{~ms}$, and finally a break of $250 \mathrm{~ms}$ followed by the presentation of the standard. The standard was presented as a blue square $4.5 \mathrm{~cm} \times 4.5 \mathrm{~cm}$ on a white background. Following the presentation of the standard there was a delay, the duration of which was drawn from a uniform distribution ranging from 2,000-5,000 ms. Following the presentation of the standard participants were instructed to reproduce the duration of the standard by terminating presence of a blue square on the screen by pressing Button 1 on a serial response box. A blue square would then appear until the participant terminated it. Participants were then instructed that a new standard was to be presented. No 
performance feedback was provided. Participants received all trials in one experimental session lasting approximately 25 minutes.

The standard was presented as a blue square $4.5 \mathrm{~cm} \times 4.5 \mathrm{~cm}$ on a white background. In order to check that the results of Experiment 2 were not contaminated by a reaction time ceiling effect for the shortest durations we changed the standard durations to the following: $401 \mathrm{~ms}, 454 \mathrm{~ms}, 512 \mathrm{~ms}$, $562 \mathrm{~ms}, 599 \mathrm{~ms}, 615 \mathrm{~ms}, 666 \mathrm{~ms}, 702 \mathrm{~ms}, 747 \mathrm{~ms}$, $803 \mathrm{~ms}, 871 \mathrm{~ms}, 923 \mathrm{~ms}, 986 \mathrm{~ms}, 1,101 \mathrm{~ms}$, or $1,141 \mathrm{~ms}$.

\section{Results}

Data were excluded from analysis when the reproduction was greater than 2 standard deviations above or below the mean. In total data from 20 of the 765 trials were excluded.

Figure 4 shows mean reproductions for each of the three testing conditions (1, 3, or 5 presentations of the standard). Comparison of performance on the three testing conditions (1, 3, or 5 presentations) shows that mean reproductions were similar in all three testing conditions.

Linear regressions were carried out on individual participants' data, giving slope and intercept values for each of the three testing conditions. A repeated measures ANOVA compared intercept

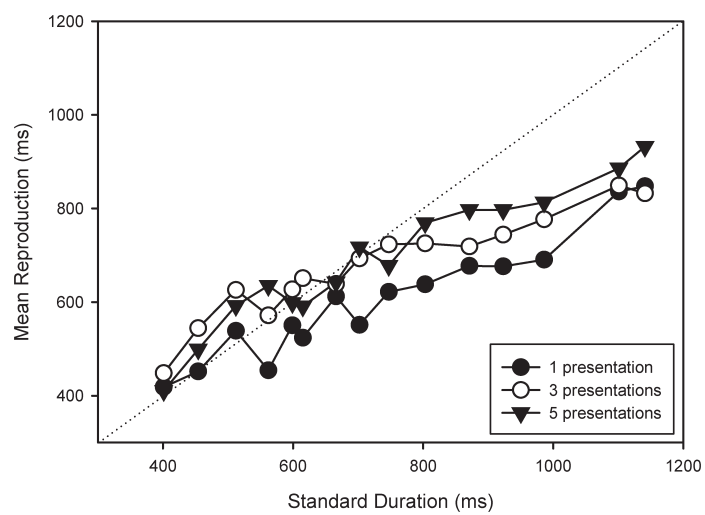

Figure 4. Mean reproductions from Experiment 3, plotted against stimulus duration in ms. Data are shown separately for 1 (filled circle), 3 (unfilled circle), and 5 (filled triangle) presentations of the standard. values and slope values from the three testing conditions. There was no significant effect of the intercept of the functions for the three conditions, $F(2,32)=1.05, p=.36$. There was also no significant effect of the slope of the functions for the three conditions, $F<1$.

\section{Discussion}

The results of Experiment 3 indicate that when a spatially and temporally relevant cue is presented before the first presentation of the standard there are no significant differences in the reproductions made in the three testing conditions. The results therefore support our theory that there is some attentional benefit to multiple presentations of a visual standard but that this benefit is lost when an attentional cue is provided prior to the presentation of the standards. The absence of an effect of the number of presentations of the standard also supports the perturbation model's account of temporal reference memory function for visual reproduction tasks. It would therefore seem that when sufficient attention is paid throughout the task the encoding and storage of stimuli from different modalities are the same. To confirm that reproductions were lengthened when a cue was provided prior to the presentation of the first standard, Experiment 4 compares reproductions when the standard is either cued and only presented once or uncued and only presented once.

\section{EXPERIMENT 4}

Experiment 4 examined whether presenting a cue prior to the presentation of the standard does actually lengthen reproductions compared to trials where no cue is presented prior to the presentation of the standard. Throughout Experiment 4 participants only received one presentation of the standard duration in each trial; on half of all trials the 3, 2, 1 countdown developed in Experiment 3 was presented prior to the presentation of standard. 


\section{Method}

\section{Participants}

A total of 14 University of Manchester students were paid $£ 5$ for participating.

\section{Procedure and apparatus}

The procedure was essentially a replication of Experiment 3; however, the standard was only presented once on each trial. On half of all trials a 3, 2, 1 countdown was employed prior to the presentation of the standard. The standard durations employed in Experiment 2 were used. All apparatus remained as in Experiments 2 and 3.

On each trial participants were informed that they were about to be presented with the standard. On cued trials, 1,500 ms before the presentation of the standard the number 3 was presented on the screen for $250 \mathrm{~ms}$. There was then a break of $250 \mathrm{~ms}$ followed by the presentation of the number 2 for $250 \mathrm{~ms}$, then a break of $250 \mathrm{~ms}$ followed by the presentation of the number 1 for $250 \mathrm{~ms}$, and finally a break of $250 \mathrm{~ms}$ followed by the presentation of the standard. The standard was presented as a blue square $4.5 \mathrm{~cm} \times 4.5 \mathrm{~cm}$ on a white background. On uncued trials no countdown was provided prior to the presentation of the standard. Following the presentation of the standard there was a delay, the duration of which was drawn from a uniform distribution ranging from 2,000-5,000 ms. Following the presentation of the standard participants were instructed to reproduce the duration of the standard by terminating the presence of a blue square on the screen by pressing Button 1 on a serial response box. A blue square would then appear until the participant terminated it. Participants were then instructed that a new standard was to be presented. No performance feedback was provided. Participants received all trials in one experimental session lasting approximately 15 minutes.

\section{Results}

Data were excluded from analysis when the reproduction was greater than 2 standard deviations above or below the mean. In total data from 5 of the 630 trials were excluded.

Figure 5 shows mean reproductions for the two testing conditions (cued or uncued) plotted against the standard duration. Examination of Figure 5 reveals that reproductions were consistently longer when an attentional cue was provided prior to the presentation of the standard. As in Experiment 2 reproductions were longer than the standard at shorter durations and shorter than the standard at longer durations, demonstrating Vierordt's law.

Linear regressions were again carried out on individual participants' data, allowing differences in the slope and intercept of the regression functions to be analysed. A repeated measures ANOVA carried out on the slope and intercept values from the individual regressions found no significant effect of cueing on intercept, $F<1$; there was, however, a significant effect on slope, $F(1,13)=6.35, M S E=0.023, p<.03$. The affect of the attentional cue on slope but not intercept is consistent with the results found in Experiment 3.

\section{Discussion}

Experiment 4 demonstrated that providing an attentional cue prior to a single presentation of a standard does indeed increase the duration of

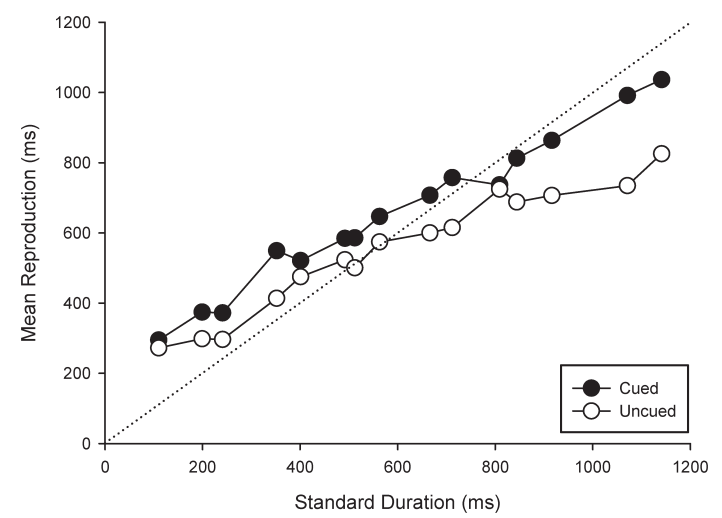

Figure 5. Mean reproductions from Experiment 4 for the two testing conditions (cued and uncued reproduction). 
reproductions in comparison with trials on which no cue was provided. This therefore confirms the findings of Experiment 3 in which is was suggested that the provision of an attentional cue prior to the first presentation of the standard would lengthen reproductions.

\section{GENERAL DISCUSSION}

The results of the experiments reported in this article and those of Jones and Wearden (2003) indicate that reference memory functions in the way as that described by the perturbation model. Experiment 1 using visual temporal generalization and the auditory condition of Experiment 2 demonstrated that increasing the number of times a standard was presented did not significantly affect responding, confirming the findings of Jones and Wearden (2003) and supporting the perturbation model account of reference memory function.

The visual condition of Experiment 2 demonstrated that reproductions were significantly shorter when the standard was only presented once than when it was presented three or five times, suggesting that the number of presentations of the standard does affect performance. No difference was found between reproductions on trials where the standard was presented three and five times. The results of the visual condition of Experiment 2 led to the suggestion that there may be some attentional benefit to multiple presentations of the standard in visual reproduction tasks. It was suggested that shorter reproductions occurred when the standard was only presented once because participants failed to accurately attend to the standard, resulting in delayed switch closure. A delay in switch closure would result in a shorter period of accumulation and therefore a shorter representation of the standard in reference memory. This theory was examined in Experiment 3, in which a countdown was provided prior to the presentation of the standard. The provision of the cue resulted in the removal of differences in the duration of reproductions when the standard was presented one, three, or five times. Experiment 3 therefore indicates that there is some attentional benefit to multiple presentations of the standard in visual reproduction. Experiment 4 confirmed the findings of Experiment 3 by demonstrating that the duration of reproductions does in fact increase when an attentional cue is provided.

One potential issue regarding our switch onset latency explanation of the shorter reproductions observed when a standard is only presented once is that switch onset/offset latencies usually manifest themselves as differences in intercept and not the slope of a function (Wearden et al., 1998). Differences in the slope of a function are normally interpreted as a result of clock speed change (Wearden et al., 1998). The performance differences observed in the visual condition of Experiment 2 resulted from the slope of the functions being significantly different across testing conditions; there were no significant differences in the intercepts of the functions. A slope effect being indicative of an attentional effect on switch operation can be accommodated if Penney's (2003) idea of an oscillating switch is accepted. Penney suggests that the switch is under attentional control but that it is more difficult for it to remain constantly closed when timing visual stimuli due to the relatively smaller attentional impact of visual stimuli; this would result in an effect that appears similar to clock speed effect. The slope effect observed in the visual condition of Experiment 2 may have occurred due to a combination of a lack of attention to the start of the stimulus and a reduced level of attention throughout the stimuli, resulting in increasingly shorter reproductions as the standard duration increases.

Taken together the results of Jones and Wearden (2003) and Experiment 1 suggest that in temporal generalization tasks reference memory operates in the same way for both modalities. Comparison of the results of Jones and Wearden and Experiment 2 suggests that the operation of reference memory for auditory stimuli is the same across temporal tasks. The visual reproduction results also suggest that when sufficient attention is paid to the standard, performance is comparable to that observed in auditory 
reproduction tasks. It would therefore seem that the perturbation model is better able to account for the pattern of responding observed in Experiments 1 to 4 than either the averaging or the sampling models. An averaging account of reference memory function is unable to accommodate these results as it would predict an improvement in performance as the number of presentations of the standard increases. Although the sampling model suggests that performance would not be affected by the number of presentations of a standard, the model is unable to accommodate fast transitions between large changes in times of reinforcement (Lejeune et al., 1997) whilst also accounting for slow transitions for small changes as in drug manipulations (Meck, 1983; also see Jones \& Wearden, 2003). The perturbation model is also more parsimonious than a sampling model, which requires several (or even dozens) of examples of the standard to be stored rather than just one.

The similarities between the way auditory and visual stimuli are encoded into reference memory across tasks suggest that the differences in auditory and visual variability are not primarily due to memory processes but are caused by some other process. The results of Experiments 1 to 4 indicate that visual stimuli require more attention to be accurately encoded, confirming suggestions by Wearden et al. (1998) that increased switch variability is the primary cause of increased variability in visual timing. Another possibility is that there may be wider bounds for the storage of visual items than auditory items in reference memory, or the transfer from short-term to long-term memory may be more variable for visual stimuli.

\section{CONCLUSIONS}

The four experiments presented in this article have demonstrated support for the perturbation model and its ability to accurately account for temporal reference memory function in modalities other than auditory and on tasks other than temporal generalization. The results indicate that the modality differences reported in the timing literature appear to be due to a difficulty in attending to or accurately perceiving visual stimuli rather than a difference in reference memory encoding between modalities.

\footnotetext{
Original manuscript received 26 February 2008 Accepted revision received 1 July 2008

First published online 10 September 2008
}

\section{REFERENCES}

Brunner, D., Fairhurst, S., Stolovitsky, G., \& Gibbon, J. (1997). Mnemonics for variability: Remembering food delay. Journal of Experimental Psychology: Animal Behavior Processes, 23, 68-83.

Church, R. M. (1984). Properties of the internal clock. In J. Gibbon \& L. G. Allan (Eds.), Timing and time perception (pp. 566-582). New York: New York Academy of Sciences.

Collier, G. L., \& Logan, G. (2000). Modality differences in short term memory for rhythms. Memory and Cognition, 28, 529-538.

Drake, C., \& Botte, M. C. (1993). Tempo sensitivity in auditory sequences: Evidence for a multiple look model. Perception and Psychophysics, 54, 277-286.

Droit-Volet, S. (2003). Alerting attention and time perception in children. Journal of Experimental Child Psychology, 85, 372-384.

Droit-Volet, S., Meck, W. H., \& Penney, T. B. (2007). Sensory modality and time perception in children and adults. Behavioral Processes, 74, 244.

Droit-Volet, S., \& Wearden, J. H. (2001). Temporal bisection in humans. Journal of Experimental Child Psychology, 80, 142-159.

Fortin, C. (1999). Short-term memory in time interval production. International Journal of Psychology, 34, 308-316.

Fortin, C., \& Breton, R. (1995). Temporal interval production and processing in working memory. Perception and Psychophysics, 57, 203-215.

Fortin, C., \& Rousseau, R. (1987). Time estimation as an index of processing demand in memory search. Perception and Psychophysics, 42, 377-382.

Fortin, C., \& Rousseau, R. (1998). Interference from short-term memory processing on encoding and reproducing brief durations. Psychological Research, 61, 269-276.

Fortin, C., Rousseau, R., Bourque, P., \& Kirouac, E. (1993). Time estimation and concurrent non- 
temporal processing: Specific interference from shortterm-memory demands. Perception and Psychophysics, 53, 536-548.

Gibbon, J. (1977). Scalar expectancy theory and Weber's law in animal timing. Psychological Review, 84, 279-325.

Gibbon, J., Church, R. M., Fairhurst, S., \& Kacelnik, A. (1988). Scalar expectancy theory and choice between delayed rewards. Psychological Review, 95, 102-114.

Glenberg, A. M., \& Jona, M. (1991). Temporal coding in rhythm tasks revealed by modality effects. Memory and Cognition, 5, 514-522.

Goldstone, S., Boardman, W. K., \& Lhamon, W. T. (1959). Intersensory comparisons of temporal judgments. Journal of Experimental Psychology, 57, 243-248.

Goldstone, S., \& Lhamon, W. T. (1974). Studies of auditory-visual differences in human time judgement: 1. Sounds are judged longer than lights. Perceptual and Motor Skills, 39, 63-82.

Grondin, S. (2005). Overloading temporal reference memory. Journal of Experimental Psychology: Human Perception and Performance, 31, 869-879.

Ivry, R. B., \& Hazeltine, R. E. (1995). Perception and production of temporal intervals across a range of durations: Evidence for a common timing mechanism. Journal of Experimental Psychology: Human Perception and Performance, 21, 3-18.

Jaskowski, P., Jaroszyk, F., \& Hogan-Jezierska, D. (1990). Temporal-order judgments and reaction time for stimuli of different modalities. Psychological Research, 52, 35-38.

Jones, L. A., \& Wearden, J. H. (2003). More is not necessarily better: Examining the nature of the temporal reference memory component in timing. Quarterly Journal of Experimental Psychology, 56B, 321-343.

Klapproth, F. (2002). The effect of study-test modalities on the remembrance of subjective duration from long-term memory. Behavioural Processes, 59, $37-46$.

Lejeune, H. (1998). Switching or gating? The attentional challenge in cognitive models of psychological time. Behavioural Processes, 44, 127-145.

Lejeune, H. (2000). Prospective timing, attention and the switch. A response to 'Gating or switching? gating is a better model of prospective timing' by Zakay. Behavioural Processes, 52, 71-76.

Lejeune, H., Ferrara, A., Simons, F., \& Wearden, J. H. (1997). Adjusting to changes in the time of reinforcement: Peak-interval transitions in rats. Journal of Experimental Psychology: Animal Behavior Processes, 17, 281-291.

McAuley, J. D., \& Kidd, G. R. (1998). Effect of deviations from temporal expectations on tempo discrimination of isochronous tome sequences. Journal of Experimental Psychology: Human Perception and Performance, 24, 1786-1800.

Meck, W. H. (1983). Selective adjustment of the speed of internal clock and memory processes. Journal of Experimental Psychology: Animal Behavior Processes, 9, 171-201.

Meck, W. H. (1988). Hippocampal function is required for feedback control of an internal clock's criterion. Behavioural Neuroscience, 102, 54-60.

Meck, W. H., Church, R. M., Wenk, G. L., \&Olton, D. S. (1987). Nucleus basalis magnocellularis and medial septal area lesions differentially impair temporal memory. Journal of Neuroscience, 7, 3505-3511.

Noulhiane, M., Pouthas, V., \& Samson, S. (in press). Is time reproduction sensitive to sensory modalities? European Journal of Cognitive Psychology.

Ogden, R. S., Wearden, J. H., \& Jones, L. A. (2008). Are memories for duration modality specific? Manuscript submitted for publication.

Olton, D. S. (1989). Frontal cortex, timing and memory. Neuropsychologia, 27, 121-130.

Penney, T. B. (2003). Modality differences in interval timing: Attention, clock speed, and memory. In W. H. Meck (Ed.), Functional and neural mechanisms of interval timing (pp. 209-234). Boca Raton, FL: CRC Press.

Penney, T. B., Gibbon, J., \& Meck, W. H. (2000). Differential effects of auditory and visual signals on clock speed and temporal memory. Journal of Experimental Psychology: Human Perception and Performance, 26, 1770-1787.

Penney, T. B., \& Tourret, S. (2005). Les effets de la modalité sensorielle sur la perception du temps [Modality effect in short interval timing]. Psychologie Française, 50, 131-143.

Rodríguez-Gironés, M. A., \& Kacelnik, A. (1999). Behavioral adjustment to modifications in the temporal parameters of the environment. Behavioural Processes, 45, 173-191.

Schulze, H. H. (1989). The perception of temporal deviations in isochronic patterns. Perception and Psychophysics, 45, 291-296.

Sebel, A. J., \&Wilsoncroft, W. E. (1983). Auditory and visual differences in time perception. Perception and Motor Skills, 57, 295-300. 
Walker, J. T., \& Scott, K. J. (1981). Auditory-visual conflicts in the perceived duration of lights, tones and gaps. Journal of Experimental Psychology: Human Perception and Performance, 7, 1327-1339.

Wearden, J. H. (2003). Applying the scalar timing model to human time psychology: Progress and challenges. In H. Helfrich (Ed.), Time and mind: II. Information-processing perspectives (pp. 21-39). Gottingen, Germany: Hogrefe \& Huber.

Wearden, J. H., Edwards, H., Fakhri, M., \& Percival, A. (1998). Why "sounds are judged longer than lights": Application of a model of the internal clock in humans. The Quarterly Journal of Experimental Psychology, 51B, 97-120.

Wearden, J. H., \& Jones, L. A. (2007). Is the growth of subjective time in humans a linear or non-linear function of real time? Quarterly Journal of Experimental Psychology, 60, 1289-1302.

Wearden, J. H., Todd, N. P. M., \& Jones, L. A. (2006). When do auditory/visual differences in duration judgements occur? Quarterly Journal of Experimental Psychology, 59, 1709-1724.

Woodrow, H. (1930). The reproduction of temporal intervals. Journal of Experimental Psychology, 13, 473-499.

Zakay, D. (2000). Gating or switching? Gating is a better model of prospective timing (a response to "switching or gating?" by Lejeune). Behavioural Processes, 52, 63-69.

Zakay, D., \& Block, R. A. (1997). Temporal cognition. Current Directions in Psychological Science, 6, 12-16. 